\title{
Revolute Quadric Decomposition of Canal Surfaces and Its Applications
}

\author{
Jinyuan $\mathrm{Jia}^{1}$, Ajay Joneja ${ }^{2}$, and Kai Tang ${ }^{2}$ \\ ${ }^{1}$ Zhuhai College of Jilin University, \\ Jinwan District, Zhuhai, P. R. China, 113001 \\ csjyjia@yahoo.com.cn \\ ${ }^{2}$ The Hong Kong University of Science and Technology, \\ Clear Water Bay, Kowloon, Hong Kong, P. R. China \\ \{Joneja, mektang\} aust.hk
}

\begin{abstract}
Surfaces subdivision is an important means for geometric computing of surfaces in CAD. This paper proposes a new quadric subdivision for canal surfaces in this paper, $R Q$-sphere decomposition, that subdivides canal surfaces as a set of truncated revolute quadric with joint spheres. Experimental results show that the RQ-sphere decomposition is better than existing methods.
\end{abstract}

\section{Introduction}

Canal surfaces are very useful for representing long thin objects, for instance, poles, 3D fonts, brass instrument or internal organs of the body in solid modeling and $\mathrm{CG} / \mathrm{CAD} / \mathrm{CAM}$. It includes natural quadrics (cylinder, cone and sphere), revolute quadrics, tori, pipes and Dupin cyclide. Therefore, some algorithms of geometric computing around canal surfaces have been developed recently [2], [3], [6], [8], [9], [10], [11], [12] and [13], in which, the decomposition schemes of canal surfaces play an important role. Although quadrilateral/triangular subdivision is a general scheme to subdivide a general surface into a quadrilateral/triangular mesh, however, it is too general so that it becomes inefficient when applied to canal surfaces directly. Canal surfaces possess some good geometric properties and should have more efficient decomposition scheme than triangulation. This paper presents new revolute quadric decomposition scheme for canal surfaces.

Besides quadrilateral or triangular decomposition, the three decomposition schemes have been given specially for canal surfaces: (1) circle decomposition, (2) cone-sphere decomposition and (3) cyclide decomposition. Circle decomposition subdivides a canal surface as a densely set of characteristic circles along its spine curve. It reduces some geometric computations of canal surfaces to the corresponding ones on circles. Several geometric problems of canal surfaces have been given in [5], [6], [8] and [10] based on circle decomposition. Cone-sphere [11] samples a set of spheres along its spine curve and joint each two adjacent sampling spheres with a common tangential truncated cone, thus, a canal surface can be approximated with a set of alternating truncated cones and spherical strips. However, it has two drawbacks: (1) at the high precision, a very dense set of cone-sphere pairs 
are required, and (2) when the shape of canal surface bends at a big curvature or torture, two neighboring truncated cones may intersect each other, see Fig 3(a), that is called self-intersection. Cyclide decomposition [13] subdivides a canal surface as a group of bounded Dupin cyclide pairs. This is a natural decomposition scheme for canal surfaces. However, cyclide is a degree-four surface, its related geometric computing have no closed form solutions, thus, it does not reduce geometric computing complexity of canal surfaces.

Quadrics are the lowest order curved surfaces. They have good parametric and implicit forms. Some related geometric computations are relatively easy and simple. Therefore, how to construct a piecewise quadric net of a surface becomes important in CAGD and some methods have been proposed on constructing a piecewise quadric spline to approximate an arbitrary surface, see [1], [4], [14] and [15]. However, while suitable for general surfaces, the aforementioned schemes become inefficient or too complicated when they are directly applied to special surfaces. It is natural to explore the specific quadric decomposition schemes for canal surface. It has some useful geometric properties that can just be exploited to develop more efficient quadric decomposition schemes.

In this paper, we propose a revolute quadric-spherical stripes decomposition for a regular canal surfaces, which can avoid the two problems of the cone-sphere scheme above. The main idea is (1) to sample a set of spheres along the spine curve appropriately and (2) construct a common tangential revolute quadric between each pair of adjacent sampling spheres, instead of the common tangential truncated cone, to approximate the canal surface. We note that the revolute quadric-spherical sphere (hereafter RQ-sphere) scheme is a natural extension of cone-sphere decomposition scheme.

\section{Preliminary}

We assume that a canal surface with a regular spine curve $\mathbf{C}(t)$ and a rational radius function $R(t)$ is valid, $\left\|\mathbf{C}^{\prime}(t)\right\| \neq 0$, and $\left\|\mathbf{C}^{\prime}(t)\right\|^{2}-R(t)^{2}>0 . \mathbf{C}_{k}(t)$ (see Fig. 1) and $R_{k}(t)$ are the center point and the radius of its characteristic circle. Then its equation $K(t, \theta)$ can be given as the following Frenet form $(\mathbf{T}(t), \mathbf{N}(t), \mathbf{B}(t))$ of $\mathbf{C}(t)$, see [8] and [9].

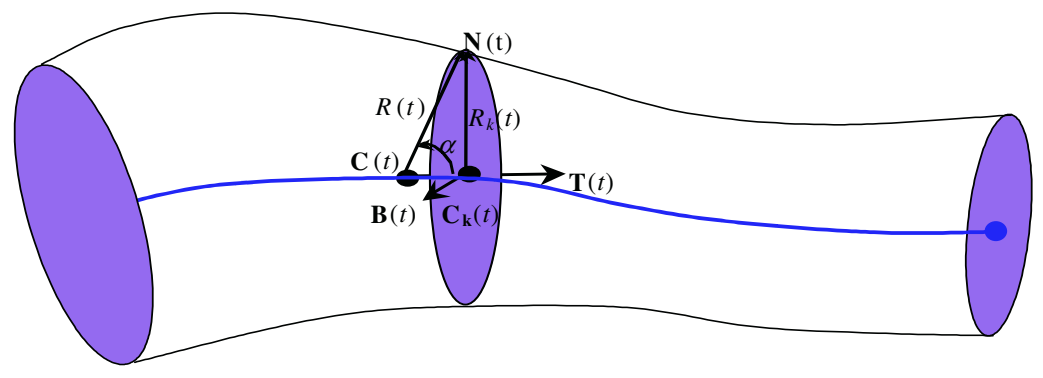

Fig. 1. The characteristic circle of canal surfaces 


$$
\mathbf{K}(t, \theta)=\mathbf{C}_{\mathbf{k}}(t)+R_{k}(t)(\cos (\theta) \mathbf{N}(t)+\sin (\theta) \mathbf{B}(t)) .
$$

where

$$
\begin{aligned}
& \mathbf{T}(t)=\left(x^{\prime}(t), y^{\prime}(t), 0\right), \quad \mathbf{B}(t)=(0,0,1), \\
& \mathbf{N}(t)=\frac{\left(-y^{\prime}(t), x^{\prime}(t), 0\right)}{\sqrt{x^{\prime}(t)^{2}+y^{\prime}(t)^{2}}}, \quad \mathbf{C}_{\mathbf{k}}(t)=\mathbf{C}(t)-R(t) R^{\prime}(t) \frac{\mathbf{C}^{\prime}(t)}{\left\|\mathbf{C}^{\prime}(t)\right\|^{2}}, \quad R_{k}(t)=R(t) \frac{\sqrt{\left\|\mathbf{C}^{\prime}(t)\right\|^{2}-R^{\prime}(t)^{2}}}{\left\|\mathbf{C}^{\prime}(t)\right\|}
\end{aligned}
$$

\section{RQ-Sphere Decomposition of Canal Surfaces}

In this section, we address three problems: $(a)$ how to construct a simple RQ-sphere fitting a canal surface for two given sampling spheres on the spine curve, $(b)$ how to avoid self-intersection of two neighboring RQs, and (c) how to optimize local fitting between a RQ-Sphere and the canal surface.

\subsection{Construction of Local RQ-Sphere Fitting}

We construct a local coordinate system with the centers of two sampling spheres $S_{l}$ and $S_{2}$ at $(0,0,0)$ and $(c, 0,0)$ respectively as shown in $(a)$ of Fig. 2 , and the revolute axis of the common tangential revolute quadric of $S_{1}$ and $S_{2}$ as $x$-axis. Then

Sphere $S_{1}$ :

$$
x^{2}+y^{2}=r^{2}
$$

Sphere $S_{2}: \quad(x-c)^{2}+y^{2}=r^{2}$,

Revolute quadric $R Q: \quad A x^{2}+y^{2}+D x+F=0$.

By substituting Eq. (2) into Eq. (4), we get a quadratic equation $(A-1) x^{2}+D x+$ $\left(F+r^{2}\right)=0$, since the RQ is tangential to $S_{l}$, its determinant must be zero:

$$
\Delta_{1}=D^{2}-4(A-1)\left(F+r^{2}\right)=0 .
$$

Similarly by substituting Eq. (3) into Eq. (4), we get equation $(A-1) x^{2}+(D+2 c) x+$ $\left(F+R^{2}-c^{2}\right)=0$, its determinant also should be zero, since $R Q$ is tangential to $S_{2}$ :

$$
\Delta_{2}=(D+2 c)^{2}-4(A-1)\left(F+R^{2}-c^{2}\right)=0 .
$$

From Eq. (5) and Eq. (6), we can obtain the other two coefficients $D$ and $F$ as functions of $A$ :

$$
D=\frac{A\left(R^{2}-r^{2}-c^{2}\right)+\left(r^{2}-R^{2}\right)}{c}, \quad F=\frac{D^{2}}{4(A-1)}-r^{2} .
$$

As shown in Fig. 2, there are totally four cases of the shape of RQ: common tangential hyperboloid, ellipsoid, paraboloid and truncated cone, depending on the value of the coefficient $A$. Let us analyze the impact of $A$ on the shape of common tangential revolute quadric. 
1. If $A=0$, Eq. (4) is reduced to $y^{2}+D x+F=0$;

2. If $A \neq 0$, Eq. (4) can be transformed into

$$
A\left(x+\frac{D}{2 A}\right)^{2}+y^{2}=G
$$

where

$$
G=\frac{D^{2}}{4 A}-F=\frac{D^{2}}{4 A}-\frac{D^{2}}{4(A-1)}+r^{2}=D^{2} \frac{-1}{4 A(A-1)}+r^{2}
$$

The $x$-coordinates of the tangent points on the two end spheres $S_{1}$ and $S_{2}$ can be written as:

$$
T_{x 1}=\frac{-D}{2(A-1)}, \quad T_{x 2}=\frac{-(D+2 c)}{2(A-1)}, \quad T_{x 2}-T_{x 1}=\frac{c}{1-A}
$$
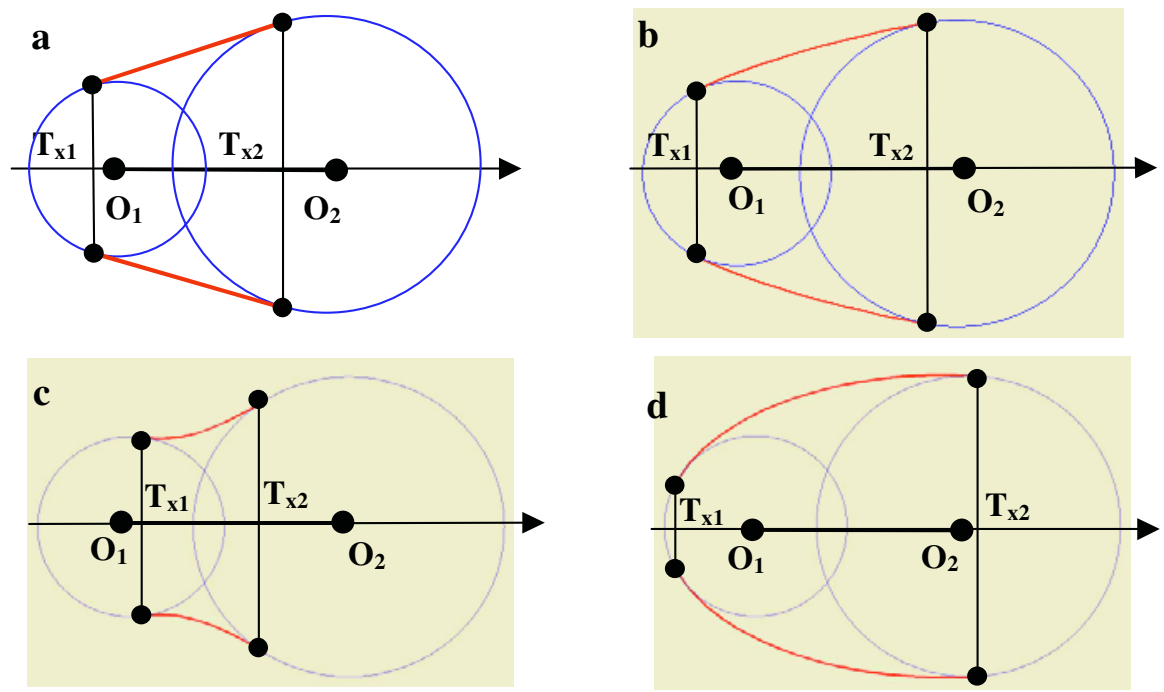

Fig. 2 Four types of RQ-Sphere fitting scheme: $\boldsymbol{a}$. Cone-sphere fitting, $\boldsymbol{b}$. Paraboloid-sphere fitting, $\boldsymbol{c}$. Hyperboloid-sphere fitting, $\boldsymbol{d}$. Ellipsoid-cone fitting

Practically, we require that $T_{x 1} \leq T_{x 2}$; thus implies $A<1$, since $c>0$. Furthermore, we can classify the scope of $A$ as follows:

1. If $0<A<1, G>0$, the corresponding RQ of Eq. (4) is an ellipsoid;

2. If $G=0$, Eq. (4) represents a truncated cone. So cone-sphere is a special case of RQ-sphere decomposition;

3. If $A<0$ and $G>0$, Eq. (4) represents a hyperboloid of one leaf.

4. If $A<0$ and $G<0$, Eq. (4) represents a hyperboloid of two leaves. 


\subsection{Overlapping Avoidance of Two Adjacent RQs}

When the curvature of the spine curve changes acutely in cone-sphere fitting, two adjacent fitting truncated cones $T C_{1}$ and $T C_{2}$ for three specified sampling spheres may intersect each other as shown in Fig. 3, it is noted as self-intersection. However, two fitting revolute quadrics can avoid such self-intersections by adjusting the coefficient $A$ of two fitting $R Q$ s appropriately. That introduces two questions: (1) how to judge such self-intersection of two fitting $R Q \mathrm{~s}$; and (2) how to the best $A$ for optimal RQsphere fitting to canal surface. To the first question, we give a theorem, the necessary and sufficient condition for self-intersection determination. To the second question, we will address it in the nest subsection.
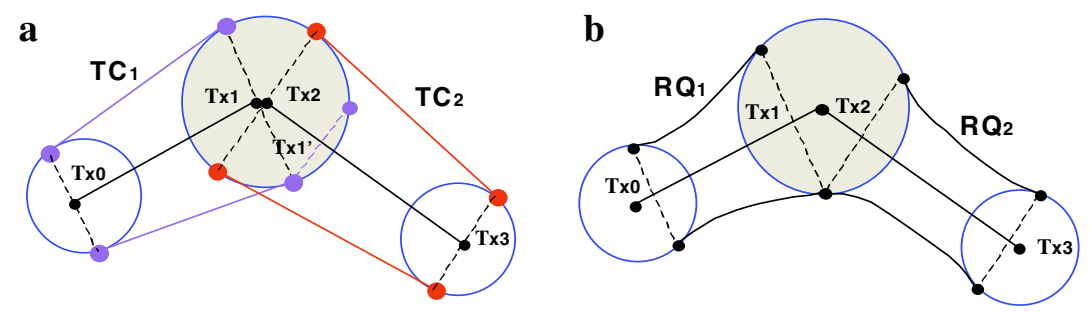

Fig. 3. Avoiding self-intersection of RQ-Sphere fitting, $\boldsymbol{a}$. Self-intersection of cone-sphere fitting, $\boldsymbol{b}$. No self-intersection of RQ-Sphere fitting
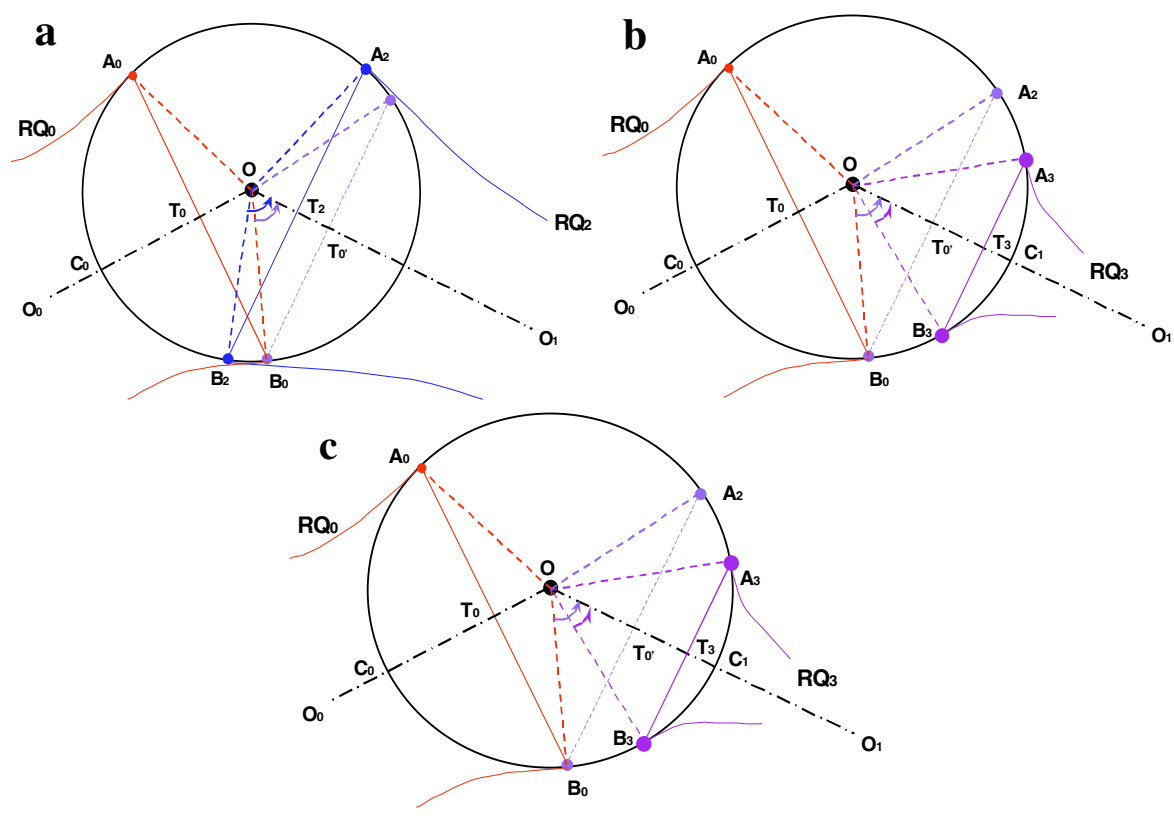

Fig. 4. Three cases of two adjacent fitting $R Q \mathrm{~s}: a$. Tangential case of two adjacent RQs, $b$. Overlapping case of two adjacent RQs, $c$. Separated case of two adjacent RQs 
Algebraically, determining intersections of two RQs requires solution of quartic equation in general. Here, we give a geometric method to solve it. The overlapping of two RQs and their coherent sphere $\mathrm{O}$ in $3 \mathrm{D}$ are equivalent to the corresponding problems of the chords $A_{0} B_{0}$ and $A_{1} B_{1}\left(A_{2} B_{2}\right.$ or $A_{2} B_{2}$, as shown in $(a),(b)$ and $(c)$ of Fig. 4 respectively) in 2D. That concludes the following theorem.

Theorem 1. Two adjacent revolute quadrics $\mathrm{RQ}_{1}$ and $\mathrm{RQ}_{2}$ have the common joint sphere, they overlap each other if and only if two corresponding chords $A_{0} B_{0}$ and $A_{l} B_{l}$ intersect each other, furthermore, if and only if $\left\|\mathrm{OT}_{0},\right\|>\left\|\mathrm{OT}_{1}\right\|$ holds.

It is much simpler than algebraic method. The proof is trivial and omitted here due to limited space.

\subsection{Locally Optimal RQ-Sphere Fitting}

By sampling a canal surface $x=x(u, v), y=y(u, v)$ and $z=z(u, v)$ with $\left(u_{i}=u_{0}, u_{1}\right.$, $\ldots \ldots, u_{m}$ and $v_{i}=v_{0}, v_{l}, \ldots \ldots, v_{n}$, the least square fitting method is employed to determine the best value for $A$ in the sense of minimizing the overall sum of square distances of the sampling points $\left(x_{i j}, y_{i j}, z_{i j}\right)$ to the fitting RQ-sphere pairs, which can be represented as:

$$
\min _{A, D, F} f(A, D, F)=\sum_{i, j}\left(A x_{i j}^{2}+y_{i j}^{2}+z_{i j}^{2}+D x_{i j}+F\right)^{2}
$$

By differentiating (27) with regards to $A$, we get

$$
\min f_{A}^{\prime}(A, D, F)=2 \sum_{i, j}^{N}\left(A x_{i j}^{2}+y_{i j}^{2}+z_{i j}^{2}+D x_{i j}+F\right)\left(x_{i j}^{2}+D_{A}^{\prime} x_{i j}+F_{A}^{\prime}\right)
$$

By substituting the following formulae:

$$
D=m A+n, D_{A}^{\prime}=m, F=\frac{(m A+n)^{2}}{4(A-1)}-r^{2}, F_{A}^{\prime}=\frac{2(m A+n) m(A-1)-(m A+n)^{2}}{4(A-1)^{2}}=k_{0}+\frac{k_{2}}{4(A-1)^{2}},
$$

where $k_{0}=m^{2} / 4$ and $k_{2}=(m-n)^{2}$, into (11), we obtain an optimization equation about $A$ as follows:

$$
\begin{array}{r}
f^{\prime}(A)=2 \sum_{i, j}^{N}\left(A x_{i j}^{2}+y_{i j}^{2}+z_{i j}^{2}+(m A+n) x_{i j}+\frac{(m A+n)^{2}}{4(A-1)}-r^{2}\right)\left(x_{i j}^{2}+m x_{i j}+k_{0}+\frac{k_{2}}{4(A-1)^{2}}\right) \\
=M_{0} A(A-1)^{3}+M_{1}(A-1)^{3}+M_{2}(m A+n)(A-1)^{3}+M_{0}(m A+n)^{2}(A-1)+ \\
+M_{3} k_{2} A(A-1)+M_{4} k_{2} A(A-1)+M_{5}(m A+n) k_{2}+(m A+n)^{2} k_{2}=0
\end{array}
$$

where

$$
\begin{aligned}
& M_{0}=\frac{16}{N} \sum_{i, j}^{\mathrm{N}} x_{i j}^{2}\left(x_{i j}^{2}+m x_{i j}+k_{0}\right), \quad M_{1}=\frac{16}{N} \sum_{i, j}^{\mathrm{N}}\left(y_{i j}^{2}+z_{i j}^{2}-r^{2}\right)\left(x_{i j}^{2}+m x_{j}+k_{0}\right), M_{2}=\frac{16}{N} \sum_{i, j}^{\mathrm{N}} x_{i j}\left(x_{i j}^{2}+m x_{i j}+k_{0}\right), \\
& M_{3}=\frac{4}{N} \sum_{i, j}^{\mathrm{N}} x_{i j}^{2}, M_{4}=\frac{4}{N} \sum_{i, j}^{\mathrm{N}}\left(x_{i j}^{2}+m x_{i j}+k_{0}\right), M_{5}=\frac{4}{N} \sum_{i, j}^{\mathrm{N}} x_{i j}
\end{aligned}
$$

Actually, Eq. (13) is a quartic equation and it has closed form solutions to $A, D$ and $F$ for a locally optimal RQ-sphere fitting canal surfaces. 


\section{Illustrative Examples}

We implemented both cone-sphere and RQ-sphere decomposition of canal surfaces for comparison. Three examples are illustrated in Fig. 5, the spine curve and radius of them are represented by cubic Bezier curve in Examp2 and Examp3. The performance data in Table 1 shows that, for the same canal surface, the ratio of the required RQsphere pairs $v s$. cone-sphere pairs nearly approaches $1: 2$. We also found that when the tolerance becomes very tiny value or the curvature of the spine curve big enough, cone-sphere results in local self-intersection unavoidably. However, RQ-sphere can avoid such local self-intersection very well.
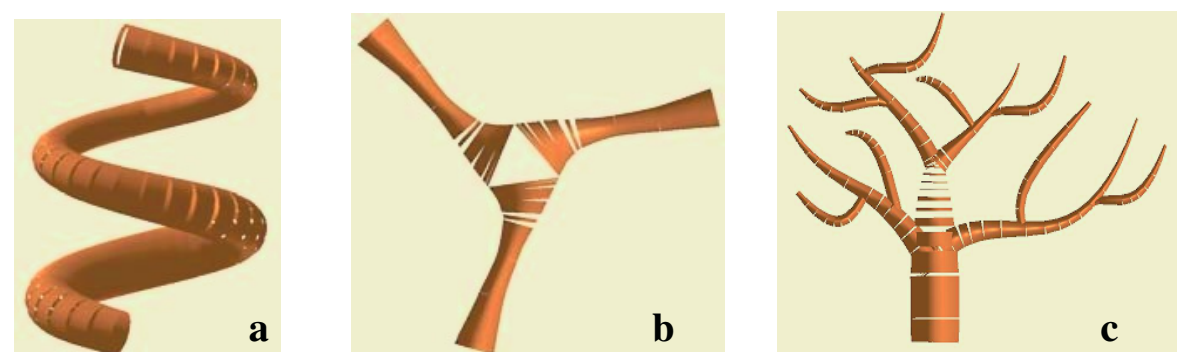

Fig. 5. Applications of RQ-sphere decompositions to canal surfaces

Table 1. Performance data of cone-sphere vs. RQ-sphere for fitting canal surfaces

\begin{tabular}{lcc}
\hline \# of Quadric-Sphere Pairs & Cone-sphere & RQ-sphere \\
\hline Example 1 at $0.5 \times 10^{-5}$ & 235 & 175 \\
Example 2 at $0.5 \times 10^{-4}$ & 1373 & 636 \\
Example 3 at $0.5 \times 10^{-3}$ & 2123 & 768
\end{tabular}

\section{Conclusions}

Compared to the cone-sphere scheme, (1) RQ-Sphere can fit canal surfaces more flexibly to avoid self-intersection and yield visually smother rendering; (2) it has one degree-of-freedom to allow optimal approximation of canal surfaces, and (3) it only requires fewer fitting components at the same approximation quality. The revolute quadric decomposition for surfaces of revolution by authors previously in [7] is a special case of RQ-sphere decomposition for canal surfaces. The following works can be extended along this paper:

1. Reconstruction of boundary canal surfaces from skeleton curves by using MAT technique becomes important. The reconstruction process in [2] and [3] (for one branch of the MAT) is exactly the definition of canal surface, (the medial axis is the spine curve $\mathrm{C}(t)$, the resulting canal surface is the boundary of reconstructed volume) Practically, the medial axis is given by a sequence of discrete sampling 
points. In fact, the process of Gelston and Dutta's reconstruction is cone-sphere fitting. Thus, our RQ-sphere can be extended to such reconstruction directly.

2. RQ-sphere decomposition can reduce the intersection computing problems of canal surfaces (ray/canal, plane/canal and canal/canal) to the corresponding problems of RQs. Also, other geometric computing problems of canal, i.e. isophote, silhouette, and so on, can be revisited in the new perspective of RQsphere decomposition.

Although RQ-sphere decomposition is better than cone-sphere decomposition, it still requires rather huge number of RQ-sphere pairs to approximate canal surfaces at the high precision, so more efficient quadric decomposition for canal surfaces and even other surfaces is our existent goal in the long run.

\section{References}

1. Bangert, C. and Prautzsch, H.: Quadric Spline. In: CAGD, Vol. 16, (1999) 497-515.

2. Ferly, E., Gascuel C. M-P, Attali D.: Skeletal Reconstruction of Branching Shapes. In: Implicit Surfaces'96, Eindhoven, Netherlands, October, (1996) 127-142.

3. Gelston S. M. and Dutta D.: Boundary Surface Recovery from Skeleton Curves and Surfaces". In: CAGD, Vol. 12, (1995) 27-51.

4. Guo B.: Quadric and Cubic Bitetrahedral Patches". In: The Visual Computer, Vol. 7, (1995) 253-262.

5. Heo H. S., Hong S. J., Seong J. K. and Kim M. S.: The Intersection of Two Ringed Surfaces and Some Related Problems". In: Graphical Model, Vol. 63, (2001) 228-244.

6. Johnstone J.: A New Intersection Algorithm for Cyclides and Swept Surfaces Using Circle Decomposition. In: CAGD, Vol. 10, (1993) 1-24.

7. Jia J. Y., Baciu G. and Kwok K. W.: Quadric Decomposition for Computing The Intersections of Surfaces of Revolution. In Graphical Models, Vol. 55, (2004) 363-383.

8. Kim K.-J. and Lee I.-K.: The Perspective Silhouette of A Canal Surface. In: Computer Graphics Forum, Vol. 22, (2003) 15-22.

9. Kim K.-J. and Lee I.-K.: Computing Isophotes of Surface of Revolution and Canal Surface. In: CAD, Vol. 5, (2003) 215-223.

10. Kim K. J., "Minimum Distance Between A Canal Surface and A Simple Surface. In CAD, Vol. 35, (2003) 871-879.

11. Nelson Max: Cone-Spheres. In ACM SIGGRAPH Computer Graphics, Vol. 24, (1991) 59-62.

12. Nishita T. and Hohan H.: A Scan Line Algorithm for Rendering Curved Tubular Objects. In: Proceedings of Pacific Graphics, (1999) 92-101.

13. Paluszny, M. and Bühler, K.: Canal Surfaces and Inversive Geometry. In: Mathematical Methods for Curves and Surfaces II, (1998) 367-375.

14. Powell, M. J. D. and M. A. Sabin: Piecewise Quadratic Approximations on Triangles. In: ACM Transaction on Mathematical Software, Vol. 3, (1977) 316-325.

15. Sed ${ }^{1}$ erberg, T.: Piecewise Algebraic Surface Patches. In. CAGD, Vol. 2, (1985) 53-60.

1 This work was partially supported by Liaoning Educational Science and Technology Fund. 